\title{
An ultra-low radioactivity measurement HPGe facility at the Center for Underground Physics
}

\author{
Moo Hyun Lee ${ }^{1}$, Woon Gu Kang, Yeongduk Kim, Eunkyung Lee, Douglas Leonard \\ Center for Underground Physics, Institute for Basic Science (IBS), \\ 55 Expo-ro, Yuseong-gu, Daejeon, 34126, Korea \\ E-mail: mhlee at ibs.re.kr
}

\section{Gowoon Kim, Su-yeon Park}

Department of Physics, Ewha Womans University,

52 Ewhayeodae-gil, Seodaemun-gu, Seoul,03760, Korea

\section{Insik Hahn}

Department of Science Education and Department of Physics, Ewha Womans University, 52 Ewhayeodae-gil, Seodaemun-gu, Seoul,03760, Korea

\begin{abstract}
The Center for Underground Physics (CUP) at Institute for Basics Science (IBS) has been conducting and preparing a few ultra-low background rare event experiments at the YangYang underground Laboratory (Y2L). In order to keep the background levels in the experiments low enough, it is critical to screen raw materials or detector components to be used in the detectors. For the screening, a number of ultra-low radioactivity detectors are developed and installed. Among the detectors installed, we have a number of HPGe (High Purity Germanium) detectors in the Y2L for measurements of gammas from the background nuclei. Two of $100 \%$ p-type coaxial single HPGe detectors were installed and have been running from 2016 for the measurement of samples with close to $100 \%$ running time. In addition, an array of 14 HPGe detectors was installed in 2017 spring after screening the array detector materials with the single HPGe detectors for an efficient measurement of gamma rays from samples with bigger volumes. Various scintillation crystals such as $\mathrm{CaMoO}_{4}, \mathrm{Li}_{2} \mathrm{MoO}_{4}$, and $\mathrm{NaI}(\mathrm{Tl})$ have been grown with purified raw materials and tested for their radioactivity background levels. In this contribution, a summary of their developments and performances will be presented.
\end{abstract}

The 39th International Conference on High Energy Physics (ICHEP2018)

4-11 July, 2018

Seoul, Korea

\section{${ }^{1}$ Speaker}




\section{Introduction}

The CUP has been developing an ultra-low background measurement facility with HPGe detectors at the Y2L from 2013 when selected as a center of the IBS. Two major experiments of the CUP, COSINE (WIMP dark matter search) [1] and AMoRE (search for neutrino-less double beta decay of ${ }^{100} \mathrm{Mo}$ isotopes) [2], are currently running at the $\mathrm{Y} 2 \mathrm{~L}$ and their upgrades are being prepared at the same time. They are looking for rare event processes, which require ultra-low background radio-activities not only from the environments (rocks and structures) but also from their detector materials which can mimic those rare events. In order to understand the environments and screen components and raw materials for the detectors, the HPGe detectors are very effective in measuring the background levels using gammas from the decays of radioactive nuclei inside the samples. Until now, we have installed and been running two of $100 \%$ p-type coaxial HPGe detectors and an array of fourteen 70\% HPGe detectors at the Y2L. In this report, installations and operations of the three different HPGe detectors together with samplemeasurements status are reported.

\section{HPGe detectors at the CUP}

There are three HPGe detectors at the CUP currently running at the Y2L. The first $100 \%$ HPGe detector was running at the Y2L from the time before 2013. The second $100 \%$ one was installed later and has been measuring samples from 2016. The array was installed in early 2017 with a quite complicated shield structure in a dedicated room and has been running since then.

\subsection{Two Single Coaxial HPGe detectors}

The two 100\% HPGe detectors, named as CC1 and CC2 (Canberra Coaxial \#1 and \#2) and located at a room at A5 tunnel in the Y2L, are shown in Fig. 1 with their shield structures and liquid nitrogen (LN) dewars $[3,4]$. The new $\mathrm{CC} 2$ has a glove-box type sealing to avoid radon contamination during the time when samples are changed. In addition to the sealing, $\mathrm{N}_{2}$ gases from LN bottles are being flushed into the acrylic box volumes constantly.
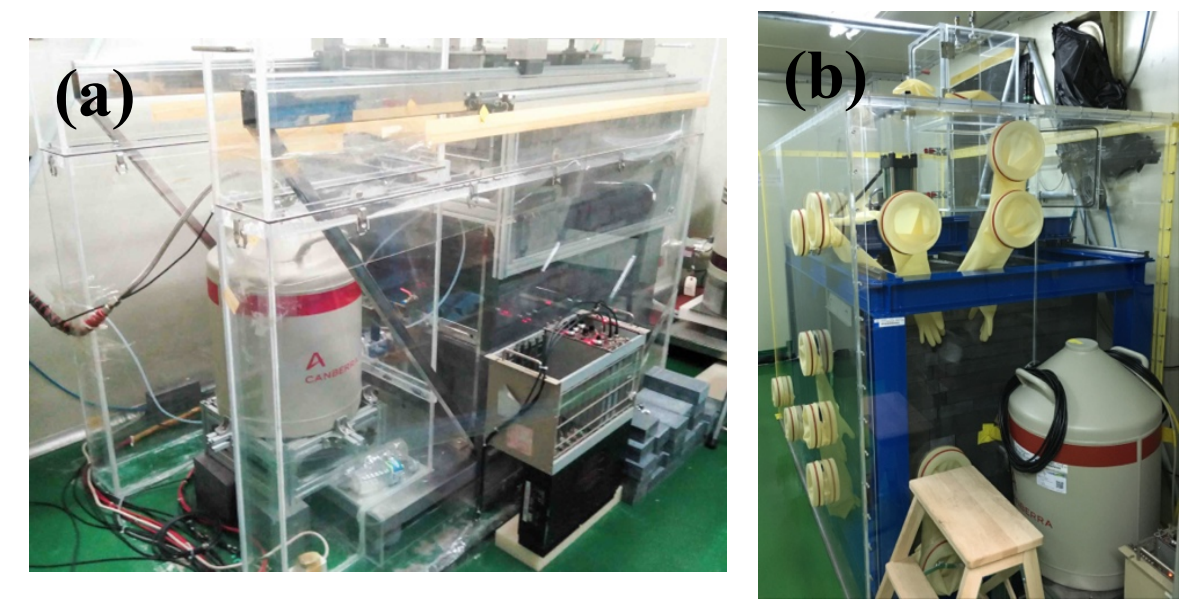

Figure 1: CC1 with shield structure and LN dewar in an acrylic box sealing (a), CC2 in a similar configuration (b).

Figure 2 shows background spectra of the two detectors during time periods when the detectors were being tuned. The $\mathrm{CC} 2$ shield was modified to improve its background as shown in Fig. 2 (b). Currently, the two background levels are similar to each other. 
For the measurements of samples, about a week was an average time for average background level samples. In addition to the samples, an experiment for searching high energy gammas is reported in this proceeding [5].
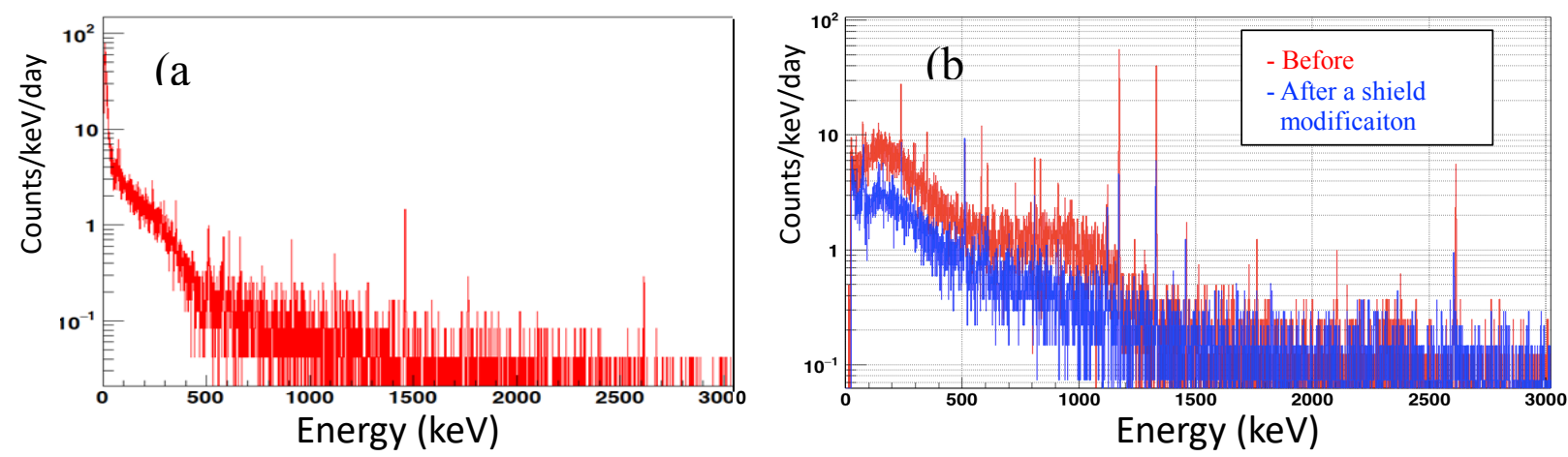

Figure 2: Background spectra of CC1 (a) and CC2 (b) before (red) and after (blue) a shield improvement.

\subsection{An array of fourteen HPGe detectors}

There is an array of fourteen $70 \%$ HPGe detectors [6] with both the top and bottom arrays have seven detectors each as shown in Fig. 3. The samples can be placed not only in the gap between the top and bottom array with adjustable height but also in the volume around the detectors to increase the detection sensitivity by having more sample weight in a measurement.

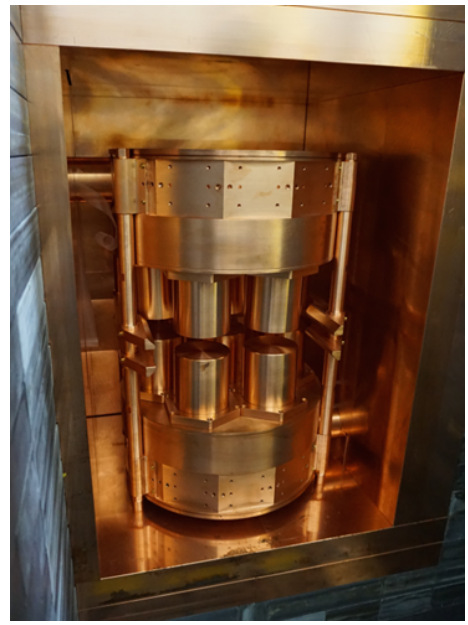

Figure 3: The array detector when the shield was open. The gap between the top and bottom array is where the samples to be placed.
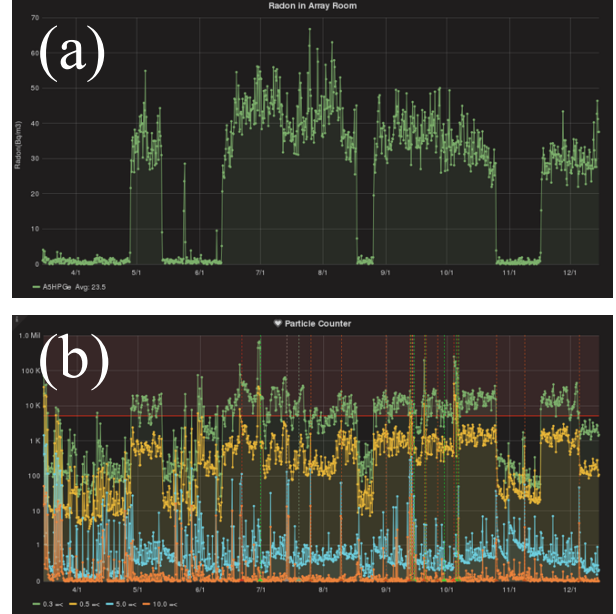

Figure 4: Radon level (a) and particle counts (b) being monitored in the array room.

The array room's main entrance door is sealed with mylar sheets to prevent the outside air coming into the room as much as possible to decrease radon contamination. Figure 4 shows monitoring histograms of radon level and particle counts (dust) in the array room for about 9 months. Radon levels in the room are from 30 to $50 \mathrm{~Bq} / \mathrm{m}^{3}$, which is reasonably low as a natural underground environment. As shown in Fig. 4 (a), the radon level went down to zero whenever the radon reduction system in the tunnel was running from time to time. Also the particle counts in the room reduced quite a lot during the operation as shown in Fig. 4 (b). We also observed increases of particle counts whenever the array room was accessed not only for a sample change but also for a simple check-up. But the array room has been kept as a class 1000 clean room during the measurement periods. The detection volume of the array has been kept being flushed 
by boil-off $\mathrm{N}_{2}$ gas from a few $\mathrm{LN}$ bottles like the two single detectors $(\mathrm{CC} 1 \& \mathrm{CC} 2)$ with a rate of $\sim 16 \mathrm{~L} / \mathrm{min}$ when the radon reduction system is not running. The installation of the array was completed in March 2017. After a tuning period until September 2017, three different measurements (or experiments) of samples were done until recently. The measurements are being analyzed and one of them using the coincidence capability is reported in this proceeding [7].

\begin{tabular}{|c|c|c|c|c|c|}
\hline CMD \# & data file name & start date & stop date & days & projects \\
\hline$\underline{102}$ & $170117-C M D 00102 C-M o O 3$ & $1 / 17$ & $2 / 10$ & 24 & AMoRE \\
\hline$\underline{124}$ & $170427-C M O \_C M D 124$ & $4 / 27$ & $5 / 19$ & 22 & Crystal,AMoRE \\
\hline Background & 170609-CC1BG & $6 / 9$ & $6 / 26$ & 17 & HPGe \\
\hline$\underline{123}$ & 170721-CMD123-phosphor-bronze-spring & $7 / 21$ & $8 / 10$ & 20 & AMoRE \\
\hline$\underline{165}$ & ThO2 powder_2kg & $9 / 29$ & $11 / 9$ & 41 & HPGe \\
\hline Background & Background_10cmPb & $11 / 9$ & $11 / 22$ & 13 & HPGe \\
\hline 172 & 171213-CMD172_KA_0195_8inchPMT & $12 / 13$ & $12 / 15$ & 2 & LZ \\
\hline
\end{tabular}

Table 1: Entries of sample measurement record (CC1) for various experiments being prepared in the CUP.

\section{Summary}

For the rare event process search experiments being upgraded in the CUP, we have been developing an ultra-low background gamma measurement facility with three HPGe detectors up to now. After installations and improvements, the two single detectors have been running almost full time quite successfully. They are mainly for screening of raw materials and detector components as summarized in table 1 for number of entries measured by the $\mathrm{CC} 1$. The array has been taking data with three different samples for about 3 months each after the installation and tuning until Sep. 2017. The array room has been being monitored with radon and dust levels in order to keep the environment background level low. With the three detectors, we plan to continue to screen samples and to do rare decay measurements to improve their detection accuracies.

\section{References}

[1] Adhikari, G., et al., Initial performance of the COSINE-100 experiment, The European Physical Journal C 78.2 (2018): 107.

[2] Alenkov, V. et al., Technical Design Report for the AMoRE 0v $\beta \beta$ Decay Search Experiment, arXiv:1512.05957.

[3] E Sala et al., Development of an underground low background instrument for high sensitivity measurements, J. Phys.: Conf. Ser. 718 (2016) 062050.

[4] E.K. Lee et al., Measurements of detector material samples with two HPGe detectors at YangYang Underground Lab., in proceedings of International Conference on High Energy Physics PoS (ICHEP2018).

[5] G.W. Kim et al., A study on high energy gamma intensities from the 208Tl decay from a $\mathrm{ThO}_{2}$ powder sample, in proceedings of International Conference on High Energy Physics PoS (ICHEP2018).

[6] E Sala et al., Development of an underground HPGe array facility for ultra low radioactivity measurements, AIP Conference Proceedings 1672 (2015) 120001; doi: 10.1063/1.4928007.

[7] S.Y. Park et al., An enriched ${ }^{100}$ Mo powder measurement by an array of HPGe detectors, in proceedings of International Conference on High Energy Physics PoS (ICHEP2018). 